\title{
Finding Asteroid belt Analogues with WISE
}

\author{
Rahul I. Patel and Stanimir Metchev \\ Department of Physics \& Astronomy, Stony Brook University, \\ 100 Nicolls Rd, \\ Stony Brook, NY \\ 11794-3800
}

\begin{abstract}
We present the detection of stars with infrared (IR) excesses attributed to circumstellar debris disks from the WISE All-Sky Survey at the WISE 12 and $22 \mu \mathrm{m}$ bandpasses ( $W 3$ and $W 4$, respectively). Excess flux at these wavelengths is significant because it traces material in the regions of terrestrial planet formation. We searched for debris disks by cross-matching Hipparcos main sequence stars with the All-Sky Data Release from WISE and seeking excess flux at $W 3$ and $W 4$. Our sample is confined to a volume of $75 \mathrm{pc}$ around the sun, and outside the galactic plane $\left(|b|>5^{\circ}\right)$. Debris disk-bearing stars were identified as $95 \%$-confidence outliers in 2MASS/WISE color distributions, after checking for erroneous photometry and contamination from unrelated nearby objects.
\end{abstract}

Keywords. stars: circumstellar matter, statistics

\section{Introduction and Cross-Match Overview}

Numerous surveys have been conducted to search for dusty circumstellar debris disks around main-sequence stars in the last few decades by IRAS, the Spitzer Space Telescope, and ISO (e.g., Rhee et al. 2007, Bryden et al. 2009, Chen et al. 2006, Su et al. 2006, Habing et al. 2001). As of now, roughly 330 debris disks hosts are known within 75 pc, detected by the excess IR thermal emission produced by the dust in the system, above the expected photospheric flux of the star. The majority of these are long wavelength excesses $(>30 \mu \mathrm{m})$, attributed to cold Kuiper-belt analogues.

Warm circumstellar dust $(100-400 \mathrm{~K})$ is more challenging to detect. Stars with this type of dust are interesting because the dust spans the frost line and habitable zone around a star. The Spitzer Space Telescope is responsible for detecting the majority of known warm dusty disks (e.g., Bryden et al. 2009, Su et al. 2009, Beichman et al. 2006), though like all pointed surveys, their limited coverage leads to biases in the overall completeness of the survey. The Wide-Field Infrared Survey Explorer (WISE; Wright et al. 2010 ) is particularly powerful in detecting these warm disks given its all-sky coverage at $W 1, W 2, W 3$ and $W 4$ bands (3.4, 4.6, 12 and $22 \mu m$ respectively). The contemporaneous coverage provides uniform 3-30 $\mu \mathrm{m}$ photometry, which allows precise estimates of the stellar photospheres and hence detections of tenuous excesses from warm dust. Our study takes advantage of this, and we provide an unbiased and complete sample of stars with excess emission at $W 3$ and $W 4$ for main-sequence Hipparcos stars within 75 pc.

\section{Excess Selection}

We initially cross-matched all Hipparcos main-sequence stars within 120 pc and outside the galactic plane $\left(|b|>5^{\circ}\right)$ to WISE sources. We used the larger 120 pc sample as a parent sample to accurately determine the photospheric behavior of stars in the WISE bands. We only used stars with a $B-V$ color between -0.1 to 1.3 mags and non-saturated 

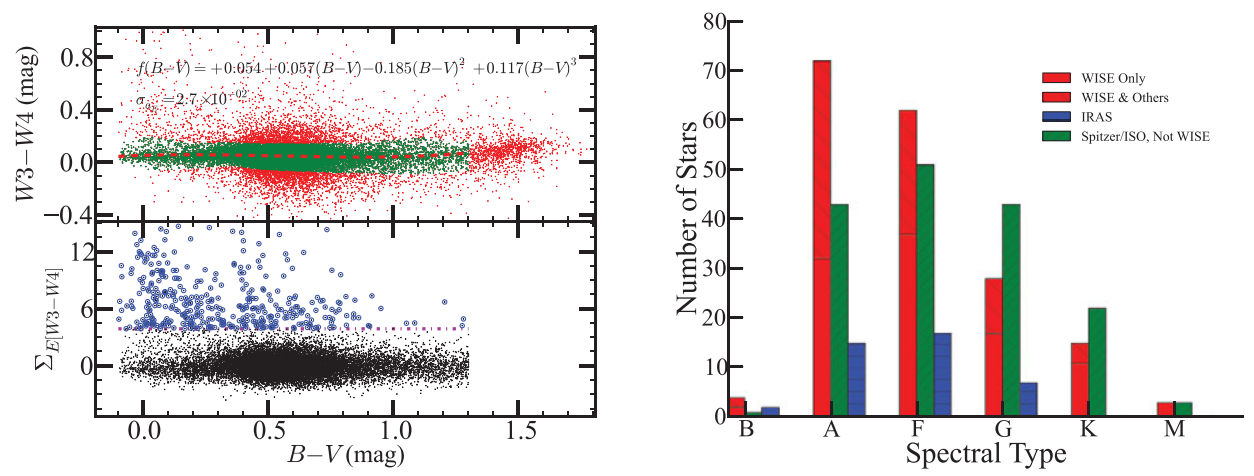

Figure 1. Left: An example $W I S E$ vs. $B-V$ color-color diagram for Hipparcos main-sequence stars within $120 \mathrm{pc}$, showing the correlation between IR and $B-V$ photospheric colors. We fit and subtract this dependence to eliminate photospheric biases in the excess. The residuals from the fit were used to assess the $95 \%$ confidence level above which stars were selected as excess candidates. Right: Spectral type distribution of stars with excesses between 10-30 $\mathrm{m}$, comparing detections of past surveys to this study.

stars in the WISE $W 2, W 3$ and $W 4$ bands. We also discarded objects with WISE flags indicating confusion, extended sources or variability. This ensured a clean sample of stars without any major systematic biases. We searched for excesses using $W 2-W 3, W 2-W 4$ and $W 3-W 4$ colors and also used 2MASS photometry for saturated stars in $W 2$ (i.e., $\left.K_{s}-W 3, K_{s}-W 4\right)$. Stars with a $95 \%$ probability of having an excess were selected from a comparison to the intrinsic WISE or $K_{s}-W I S E$ color distribution as a function of stellar $B-V$ colors. The selection process can be seen in the left panel of Figure 1. Stars were selected this way for each of the considered color combinations and their 2MASS and WISE images were visually inspected for contamination from nearby objects.

\section{Results}

We detect 184 stars within 75 pc with IR excesses at 12 or $22 \mu \mathrm{m}, 115$ of which are new detections at these wavelengths. One hundred and two of the disks are new detections altogether and thus expand the number of known debris disks within $75 \mathrm{pc}$ of the Sun by $36 \%$. We find that $14 \%$ of A stars possess warm $(\gtrsim 100 \mathrm{~K})$ excesses with $L_{\text {dust }} / L_{*} \gtrsim 10^{-4.5}$, while the incidence rate among solar type stars is $1 \%$. A breakdown of the number of stars detected to have an IR excess can be seen in the right panel of Figure 1 .

\section{References}

Beichman, C. A., et al. 2006a, ApJ, 652, 1674

Bryden, G., et al. 2009, ApJ, 705, 1226

Carpenter, J. M., et al. 2009, ApJS, 181, 197

Chen, C. H., et al. 2006, ApJS, 166, 351

Cutri, R. M., et al. 2012, Explanatory Supplement to the WISE All-Sky Data Release Products, Technical Report

Habing, H. J., et al. 2001, A\&A, 365, 545

Rhee, J. H., Song, I., Zuckerman, B., \& McElwain, M. 2007, ApJ, 660, 1556

Su, K. Y. L., et al. 2006, ApJ, 653, 675

Su, K. Y. L., et al. 2009, ApJ, 705, 314

Wright, E. L., et al. 2010, AJ, 140, 1868 